\title{
Mechanism of uncoupling protein 2-mediated myocardial injury in hypothermic preserved rat hearts
}

\author{
GAI-GE CHEN $^{1}$, JIN-BIN YAN ${ }^{1}$, XU-MING WANG ${ }^{2}$, MING-ZHI ZHENG ${ }^{3}$, \\ JIAN-PING JIANG ${ }^{4}$, XIN-MEI ZHOU ${ }^{5}$, BIN CAI $^{6}$ and YUE-LIANG SHEN ${ }^{1}$ \\ ${ }^{1}$ Department of Pathology and Pathophysiology, Zhejiang University School of Medicine, Hangzhou, Zhejiang 310058; \\ ${ }^{2}$ Department of Pharmacology, Medicine School, Taizhou University, Taizhou, Zhejiang 317000; \\ Departments of ${ }^{3}$ Pharmacology and ${ }^{4}$ Clinical Medicine, Zhejiang Medical College, Hangzhou, Zhejiang 310053; \\ ${ }^{5}$ Department of Physiology, Jiaxing University College of Medicine, Jiaxing, Zhejiang 314001; \\ ${ }^{6}$ Department of Medical Affairs, Sir Run Run Shaw Hospital, Zhejiang University School of Medicine, \\ Hangzhou, Zhejiang 310016, P.R. China
}

Received June 22, 2015; Accepted June 10, 2016

DOI: $10.3892 / \mathrm{mmr} .2016 .5436$

\begin{abstract}
In the present study, the alterations in uncoupling protein 2 (UCP2) expression following hypothermic preservation in rat hearts were investigated. Isolated rat hearts were preserved in Celsior solution for 3-12 h followed by $60 \mathrm{~min}$ of reperfusion. The cardiac function was evaluated using the Langendorff perfusion system. UCP2 and silent mating type information regulation 2 homolog 1 (SIRT1) proteins were detected by western blot analysis. The ATP production and mitochondrial reactive oxygen species (ROS) levels were assessed. Subsequent to preservation in ice-cold Celsior solution for 3-12 h, the UCP2 protein expression in rat hearts was observed to increase in a time-dependent manner. The UCP2 inhibitor genipin inhibited the hypothermic preservation-induced cardiac dysfunction, prevented a decline in ATP production induced by $9 \mathrm{~h}$ of preservation, however had no effect on the hypothermic preservation-induced increase in mitochondrial ROS levels. Compared with the control group, the SIRT1 protein expression in rat hearts reduced following hypothermic preservation. Compared with the 9-h preservation group, Celsior solution supplemented with the SIRT1 activator resveratrol (20 or $40 \mu \mathrm{mol} / \mathrm{l})$ inhibited UCP2 protein
\end{abstract}

Correspondence to: Professor Yue-Liang Shen, Department of Pathology and Pathophysiology, Zhejiang University School of Medicine, 866 Yu-Hang-Tang Road, Hangzhou, Zhejiang 310058, P.R. China

E-mail: shenyueliang@zju.edu.cn

Ms. Bin Cai, Department of Medical Affairs, Sir Run Run Shaw Hospital, Zhejiang University School of Medicine, 3 Qingchun East Road, Hangzhou, Zhejiang 310016, P.R. China

E-mail: caib@srrsh.com

Key words: heart, hypothermic preservation, mitochondria, apoptosis, uncoupling protein 2 overexpression, prevented the decline in ATP production and resulted in an improvement cardiac function. The SIRT1 inhibitor EX-527 abolished the resveratrol-induced inhibition of UCP2 overexpression and cardiac protection in the hypothermic preserved rat heart. These observations suggest that downregulation of UCP2 expression in the hypothermic preserved rat heart in part initiated the protective mechanism via the SIRT1 pathway.

\section{Introduction}

Primary graft dysfunction due to ischemia/reperfusion injury is a major cause of cardiac dysfunction and high mortality $(1,2)$. Mitochondrial dysfunction serves a crucial role in the pathogenesis of several cardiac diseases including ischemia/reperfusion injury. In response to stress, mitochondria are able to rapidly shut off the energy supply, produce vast amounts of toxic oxygen species and release a mixture of death-inducing proteins into the cellular milieu (3). Mitochondrial uncoupling proteins (UCPs), which promote proton leak across the inner mitochondrial membrane, have emerged as essential regulators of mitochondrial membrane potential, respiratory activity and reactive oxygen species (ROS) generation. UCP2 is able to reduce mitochondrial ROS generation and thereby ameliorate myocardial function by promoting mild uncoupling (4). UCPs contribute to the elevation of cardiomyocyte tolerance against hypoxia and re-oxygenation, and promote cellular resistance to oxidative stress (4). UCP2 levels have been reported to be upregulated in parallel with infarct size reduction in preconditioned hearts (5). UCP2 gene expression is increased in the left ventricle in response to chronic hypobaric hypoxia (6). In the cytoprotective hierarchy, UCP2 appears to serve a role in modulating ischemia/anoxia tolerance in heart-derived cells. Therefore, mitochondrial UCPs are necessary components of ischemia tolerance and function as components of the cellular antioxidant defense program.

The pathophysiological role of UCP2 on influencing post-operative outcomes following heart transplantation 
remains to be fully elucidated. Considering its pivotal role in the pathogenesis of the organs post-ischemia/reperfusion injury, further investigation on cardiac UCP2 activity and regulation will facilitate the development of novel and more efficient organ preservation. In the present study, the role of UCP2 following hypothermic preservation in rat hearts was investigated using the Langendorff perfusion system. Due to the fact that SIRT1 is an important regulators of UCP2 expression in numerous physiological and pathophysiological conditions, the role of SIRT1 in the alterations of UCP2 expression was additionally investigated in the current study.

\section{Materials and methods}

Animals. Male Sprague-Dawley rats ( $\mathrm{n}=88$; age, 8-10 weeks; weight, 250-300 g) were purchased from the Experimental Animal Center of Zhejiang University (Hangzhou, China) and cared for in compliance with the Guide for the Care and Use of Laboratory Animals published by the National Institutes of Health (7). Rats were housed at five rats per cage in a controlled environment at $21-23{ }^{\circ} \mathrm{C}$, with $40-60 \%$ humidity and $12 / 12-\mathrm{h}$ light/dark cycle. The rats were allowed free access to water and food. All experimental protocols were approved by the Ethics Committee on Animal Experimentation of Zhejiang University.

Reagents. Genipin, resveratrol, EX-527, ethylene glycol-bis( $\beta$-aminoethyl ether)-N,N,N',N'-tetraacetic acid (EGTA) and monoclonal mouse $\beta$-actin antibody (cat. no. A5316) were purchased from Sigma-Aldrich (St. Louis, MO, USA). The rabbit polyclonal UCP2 antibody (cat. no. 11081-1-AP) was purchased from Proteintech Group, Inc. (Chicago,IL, USA). Rabbit polyclonal SIRT1 (cat. no. sc-15404) and mouse monoclonal prohibitin (cat. no. sc-377037) antibodies were purchased from Santa Cruz Biotechnology, Inc. (Dallas, TX, USA). Krebs-Henseleit (KH) solution (pH 7.4) consisted of: $\mathrm{NaCl} 118.0 \mathrm{mmol} / \mathrm{l}, \mathrm{KCl} 4.7 \mathrm{mmol} / 1$, $\mathrm{K}_{2} \mathrm{PO}_{4} 1.2 \mathrm{mmol} / 1, \mathrm{MgSO}_{4} 1.2 \mathrm{mmol} / 1, \mathrm{NaHCO}_{3} 25.0 \mathrm{mmol} / \mathrm{l}$, $\mathrm{CaCl}_{2} 1.25 \mathrm{mmol} / 1$ and glucose $10.0 \mathrm{mmol} / \mathrm{l}$. Celsior solution (pH 7.4) consisted of: $\mathrm{NaOH} 100 \mathrm{mmol} / \mathrm{l}, \mathrm{KCl} 15 \mathrm{mmol} / \mathrm{l}$, $\mathrm{MgCl}_{2} 13 \mathrm{mmol} / 1, \mathrm{CaCl}_{2} 0.25 \mathrm{mmol} / 1$, mannitol $60 \mathrm{mmol} / \mathrm{l}$, lactobionate $80 \mathrm{mmol} / 1$, histidine $30 \mathrm{mmol} / 1$, glutamate $20 \mathrm{mmol} / \mathrm{l}$.

In vitro hypothermic heart preservation model. Male Sprague-Dawley rats were anesthetized with $60 \mathrm{mg} / \mathrm{kg}$ sodium pentobarbital (intraperitoneal). The hearts were rapidly excised and washed in ice-cold KH solution, and mounted for retrograde perfusion using a modified Langendorff method (8). Following equilibration perfusion with $\mathrm{KH}$ solution $\left(37^{\circ} \mathrm{C}\right)$ for $30 \mathrm{~min}$, the left ventricular end-diastolic pressure (LVEDP), left ventricular developed pressure (LVDP), maximal systolic velocity of left ventricular pressure $\left(+\mathrm{dP} / \mathrm{dt}_{\max }\right)$, maximal diastolic velocity of left ventricular pressure $\left(-\mathrm{dP} / \mathrm{dt}_{\max }\right)$ and heart rate were recorded as the basal value. Ice-cold Celsior solution was perfused into the aorta to induce cardiac arrest, then the rat hearts were subjected to $3-12 \mathrm{~h}$ of preservation in ice-cold Celsior solution followed by reperfusion with $\mathrm{KH}$ solution $\left(37^{\circ} \mathrm{C}\right)$ for $60 \mathrm{~min}$. Coronary flow was recorded during the equilibration and reperfusion.
Animal grouping. Rat hearts were divided into the following groups ( $\mathrm{n}=8$ ): i) Control group, not preserved in Celsior solution; ii) Celsior group, preserved in Celsior solution for 3-12 h; iii) genipin group, preserved in Celsior solution containing genipin $(10 \mu \mathrm{mol} / \mathrm{l})$ for $9 \mathrm{~h}$; iv) resveratrol group, stored in Celsior solution containing resveratrol $(10,20$ and $40 \mu \mathrm{M})$ for $9 \mathrm{~h}$; v) resveratrol + EX-527 group, stored in Celsior solution containing resveratrol $(40 \mu \mathrm{M})$ and EX-527 $(10 \mu \mathrm{mol} / \mathrm{l})$ for $9 \mathrm{~h}$; and vi) EX-527 group, stored in Celsior solution containing EX-527 $(10 \mu \mathrm{mol} / \mathrm{l})$ for $9 \mathrm{~h}$.

Isolation of mitochondria. Cardiac mitochondria were prepared from rat hearts as previously described (9). Briefly, rat ventricular myocardium was excised and homogenized in an ice-cold isolation buffer with EGTA (1 mmol/l). The homogenates were centrifuged at $1,000 \mathrm{x} \mathrm{g}$ for $10 \mathrm{~min}$ at $4^{\circ} \mathrm{C}$, then the supernatants were collected and centrifuged at $10,000 \mathrm{x}$ g for $20 \mathrm{~min}$ at $4^{\circ} \mathrm{C}$. The resulting pellet was washed by resuspension in isolation buffer without EGTA and centrifuged again at $10,000 \mathrm{x} \mathrm{g}$ for $20 \mathrm{~min}$ at $4^{\circ} \mathrm{C}$. The final pellet resuspended in the isolation buffer was used for further assay. Mitochondrial purity was assessed by western blot analysis with the mitochondrial marker prohibitin and the cytosolic marker $\beta$-actin.

Measurement of reactive oxygen species. ROS production in the cardiac mitochondria was determined using 2,7-dichlorodihydro fluorescent diacetate (DCFH-DA; Beyotime Institute of Biotechnology, Haimen, China) (10). Briefly, mitochondria (10 $\mu \mathrm{g})$ were suspended in 4-(2-hydroxyethyl)-1-piperazineethanesulfonic acid buffer and were incubated with $10 \mu \mathrm{M} \mathrm{DCFH-DA}$ at $37^{\circ} \mathrm{C}$ for $20 \mathrm{~min}$ in the darkness. The fluorescence intensity was measured using a microplate reader (Infinite M200; Tecan Group, Ltd., Männedorf, Switzerland), at excitation and emission wavelengths of $485 \mathrm{~nm}$ and $530 \mathrm{~nm}$, respectively.

Myocardial adenosine triphosphate (ATP) content measurement. Myocardial ATP content was measured using the ATP assay kit (Beyotime Institute of Biotechnology) based on the luciferin-luciferase reaction. Bioluminescence intensity was determined using a microplate reader (Infinite M200). The protein concentration of each sample was determined using the Bicinchoninic Acid Protein Assay kit (Beyotime Institute of Biotechnology). Total ATP levels were expressed as $\mathrm{nmol} / \mathrm{mg}$ protein.

Western blotting. Left ventricular myocardium was homogenized in radioimmunoprecipitation assay lysis buffer (Beyotime Institute of Biotechnology). Proteins were separated by $10 \%$ sodium dodecyl sulphate-polyacrylamide gel electrophoresis and then blotted onto a nitrocellulose membrane (Invitrogen; Thermo Fisher Scientific, Inc., Waltham, MA, USA). For the detection of proteins, the membrane was incubated with the UCP2 $(1: 1,000)$, SIRT1 $(1: 200)$ or $\beta$-actin $(1: 5,000)$ primary antibodies overnight at $4^{\circ} \mathrm{C}$, and then incubated with goat anti-mouse (sc-2005) and goat anti-rabbit (sc-2004) horseradish peroxidase-conjugated secondary antibodies (1:2,000; Santa Cruz Biotechnology, Inc.) for $1 \mathrm{~h}$ at room temperature. The proteins were visualized 
A

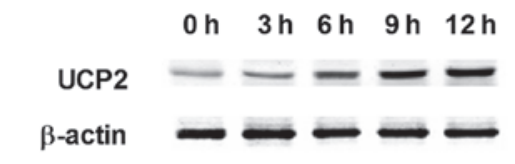

C

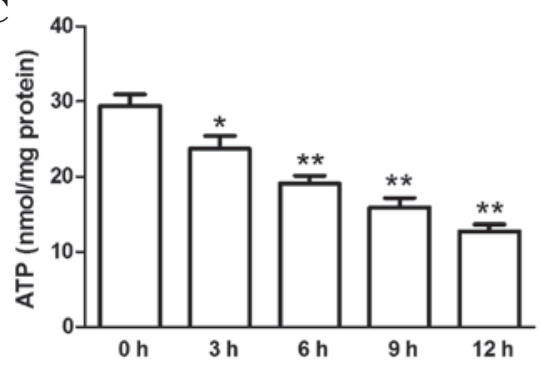

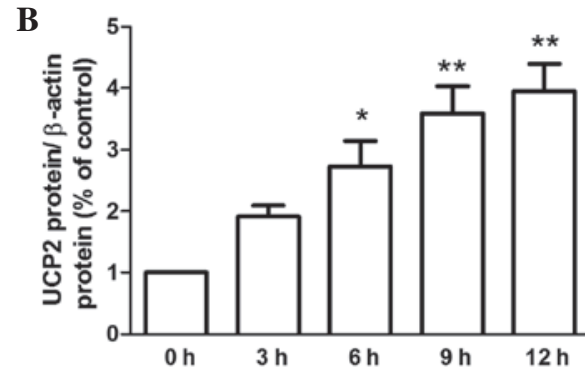

D

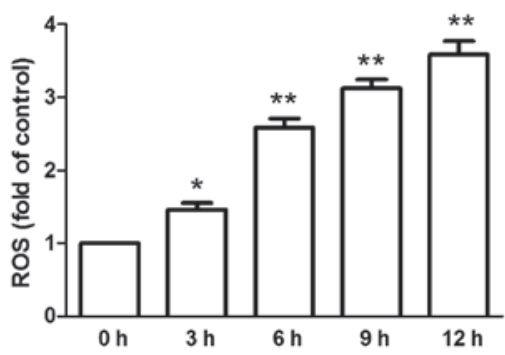

Figure 1. Expression of UCP2 protein, ATP production and ROS levels in hypothermic preserved rat hearts. (A) A representative immunoblot obtained using UCP2 and $\beta$-actin antibodies. (B) Densitometric analysis indicating the protein levels of UCP2. Data are expressed as the mean \pm standard error of three experiments. (C) ATP production, data are expressed as the mean \pm standard error, $n=8$. (D) ROS levels, data are expressed as the mean \pm standard error, $\mathrm{n}=8$. ${ }^{*} \mathrm{P}<0.05,{ }^{* *} \mathrm{P}<0.01$ vs. control group (not preserved in Celsior solution). UCP2, uncoupling protein 2; ATP, adenosine triphosphate; ROS, reactive oxygen species.

using an enhanced chemiluminescence kit (Beyotime Institute of Biotechnology). The band density was determined using Quantity One software (version 4.62; Bio-Rad Laboratories, Inc., Hercules, CA, USA) and normalized to that of $\beta$-actin.

Statistical analysis. Data were expressed as the mean \pm standard error and analyzed using a one-way analysis of variance with Newman-Keuls' post hoc test as required. $\mathrm{P}<0.05$ was considered to indicate a statistically significant difference.

\section{Results}

Alterations in UCP2 protein levels in rat hearts following hypothermic preservation. Subsequent to preservation in ice-cold Celsior solution for 3-12 h, the UCP2 protein expression levels in hypothermic preserved rat hearts were increased in a time-dependent manner (Fig. 1). The LVEDP was significantly increased, the $\mathrm{LVDP}, \pm \mathrm{dP} / \mathrm{dt}_{\max }$, heart rate and coronary flow were significantly reduced in rat hearts undergoing $9 \mathrm{~h}$ of hypothermic preservation followed by $60 \mathrm{~min}$ of reperfusion $(\mathrm{P}<0.01$; Fig. 2A-F). Celsior solution supplemented with the UCP2 inhibitor genipin reversed the hypothermic preservation-induced cardiac dysfunction $(\mathrm{P}<0.01$; Fig. 2A-F). The decline in ATP production induced by $9 \mathrm{~h}$ of preservation was also prevented by the supplement of genipin $(\mathrm{P}<0.01$; Fig. 2G). However, genipin had no effect on the hypothermic preservation-induced increase in mitochondrial ROS levels (Fig. 2H).

Effect of SIRT1 activation on hypothermic preservation-induced injury of rat hearts. Compared with the control group, the SIRT1 protein expression in rat hearts was reduced following preservation for 6-12 h (Fig. 3). The SIRT1 activator resveratrol $(20$ or $40 \mu \mathrm{mol} / \mathrm{l})$ inhibited the UCP 2 protein overexpression induced by $9 \mathrm{~h}$ of hypothermic preservation, and prevented the hypothermic preservation-induced decline in ATP production $(\mathrm{P}<0.01$; Fig. 4). Compared with $9 \mathrm{~h}$ of preservation rat hearts, Celsior solution supplement with resveratrol (20 or $40 \mu \mathrm{mol} / \mathrm{l})$ additionally prevented the hypothermic preservation-induced increase in LVEDP, improved the LVDP and $\pm \mathrm{dP} / \mathrm{dt}_{\max }$ recovery, enhanced the heart rate and coronary flow (Fig. 5).

Effect of SIRT1 inhibitor on resveratrol-induced improvement of cardiac function in hypothermic preserved rat hearts. Compared with the Celsior group, the SIRT1 inhibitor EX-527 did not significantly effect the UCP2 protein expression and cardiac function in hypothermic preserved rat hearts. However, EX-527 was able to prevent the resveratrol-induced inhibition of UCP2 overexpression ( $\mathrm{P}<0.01$; Fig. 6), and abolish the resveratrol-induced protection against cardiac dysfunction in the hypothermic preserved rat heart $(\mathrm{P}<0.01$; Fig. 7).

\section{Discussion}

Successful organ preservation is imperative to reduce the ischemia-reperfusion injury in clinical heart transplantation. Cardiac mitochondrial dysfunction is deemed as one of the key challenges limiting heart preservation. UCP2, as an inner mitochondrial membrane proton carrier that uncouples ATP synthesis, is able to facilitate proton leak into the mitochondrial matrix to promote partial uncoupled respiration; however if in excess, will facilitate mitochondrial and cellular damage. In the present study, the UCP2 protein expression in rat hearts was observed to increase in a time-dependent manner following cold preservation. The UCP2 inhibitor genipin inhibited the hypothermic preservation-induced cardiac dysfunction, prevented decline in ATP production, indicating that overexpression of UCP2 serves a negative role in myocardial ischemic injury. 
A

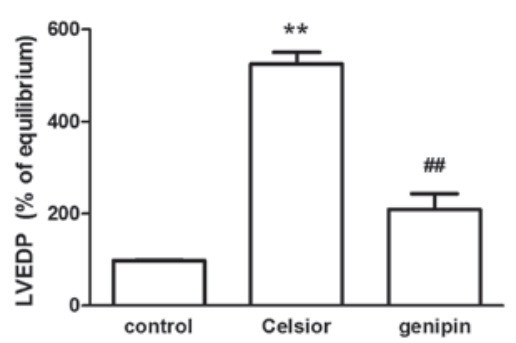

C

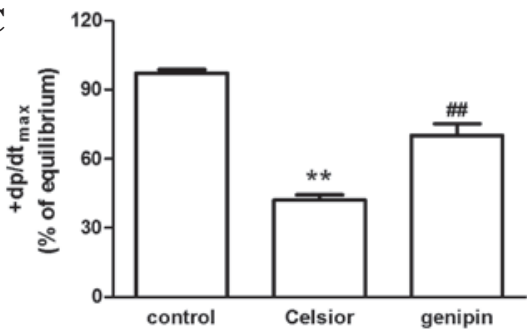

E

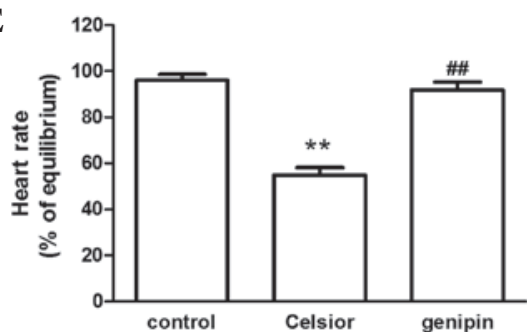

G

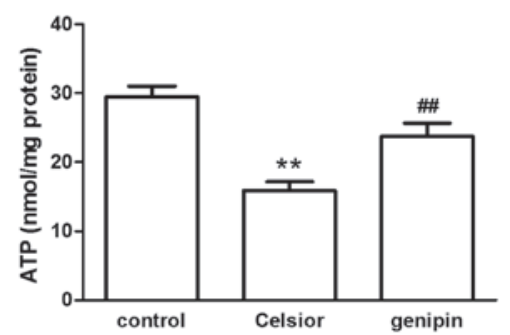

B

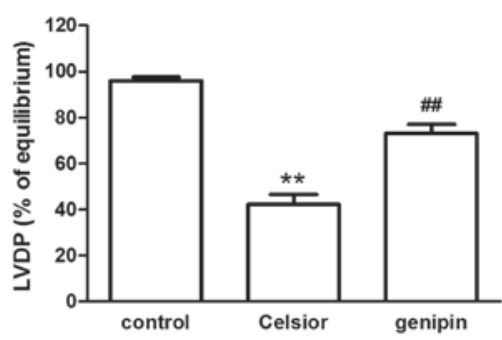

D

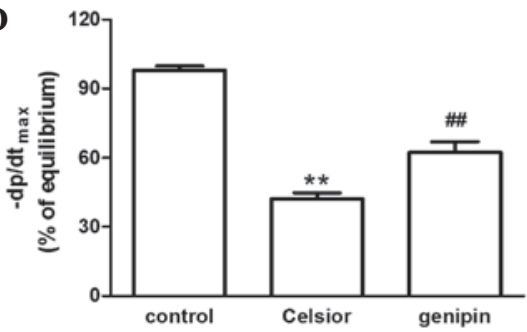

F

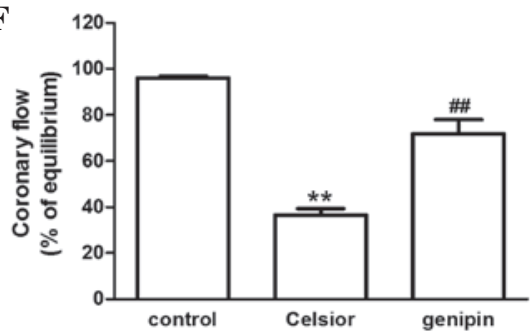

H

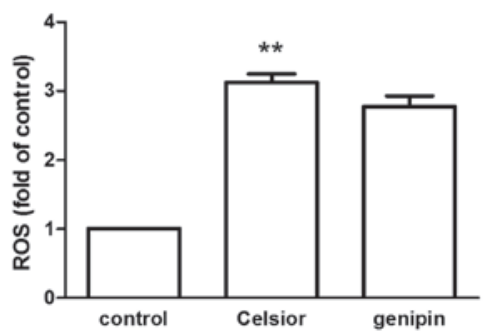

Figure 2. Effect of the UCP2 inhibitor genipin on (A) LVEDP, (B) LVDP, (C) $+\mathrm{dP}^{\mathrm{dt}} \mathrm{t}_{\max }$, (D) - $\mathrm{dp} / \mathrm{dt}_{\max }$, (E) heart rate, (F) coronary flow, (G) ATP production, and $(\mathrm{H})$ ROS level in rat hearts undergoing $9 \mathrm{~h}$ of hypothermic preservation followed by 60 min of reperfusion. Data are expressed as the mean \pm standard error, $\mathrm{n}=8 .{ }^{* *} \mathrm{P}<0.01$ vs. control group (not preserved in Celsior solution); ${ }^{\# \#} \mathrm{P}<0.01$ vs. Celsior group. UCP2, uncoupling protein 2; LVEDP, left ventricular end-diastolic pressure; LVDP, left ventricular developed pressure; $+\mathrm{dP} / \mathrm{dt}_{\max }$, maximal systolic velocity of left ventricular pressure; $-\mathrm{dP} / \mathrm{dt}_{\max }$, maximal diastolic velocity of left ventricular pressure; ATP, adenosine triphosphate; ROS, reactive oxygen species.

The exact role of upregulation of UCP2 in rat heart mitochondria remains to be determined. UCP2 has been suggested to protect cardiomyocytes against oxidative stress by dissipating the mitochondrial proton gradient and mitochondrial membrane potential, thereby reducing mitochondrial ROS generation. McLeod et al (5) reported that preconditioning in rat cardiac derived myoblasts is abolished following UCP2 depletion by RNA-interference. However, in apparent conflict with its uncoupling role, $\mathrm{UCP} 2$ has additionally been hypothesized to be essential for mitochondrial $\mathrm{Ca}^{2+}$ uptake, which has a protective action by stimulating mitochondrial ATP production. UCP2 overexpression attenuates $\mathrm{Ca}^{2+}$ overload and the production of ROS in mitochondria (11). UCP2 additionally increases sensitivity of adult rat cardiac myocytes to hypoxia-reoxygenation via ATP depletion and acidosis (12). In the present study, the UCP2 inhibitor genipin inhibited the hypothermic preservation-induced cardiac dysfunction, prevented decline in ATP production induced by $9 \mathrm{~h}$ of preservation, however had no effect on the hypothermic preservation-induced increase in mitochondrial
A
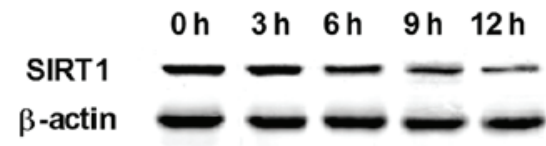

B

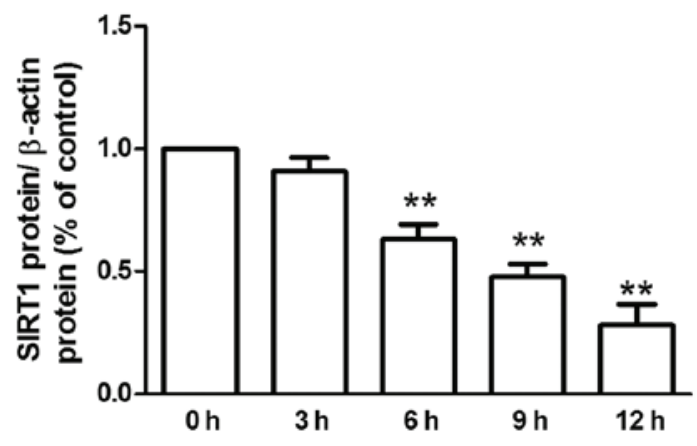

Figure 3. Expression of SIRT1 protein in hypothermic preserved rat hearts (A) Representative immunoblot obtained using SIRT1 and $\beta$-actin antibodies. (B) Densitometric analysis for SIRT1 levels. Data are expressed as the mean \pm standard error of three experiments. ${ }^{* *} \mathrm{P}<0.01$ vs. control group. Celsior group. SIRT1, silent mating type information regulation 2 homolog 1 . 
A

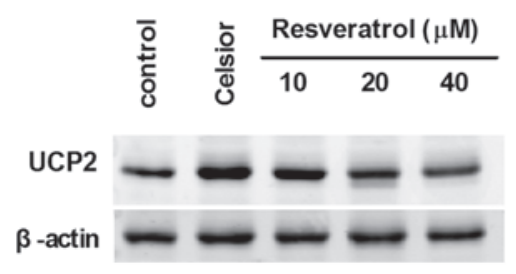

B

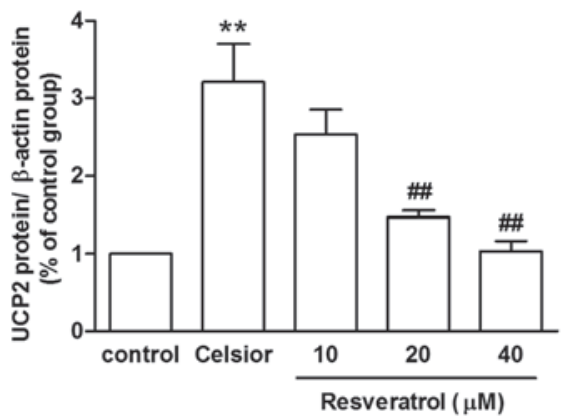

C

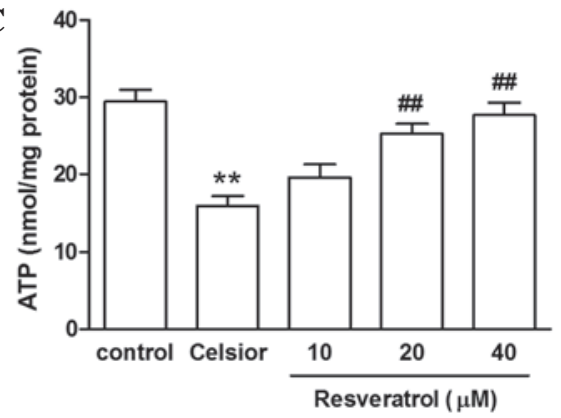

Figure 4. Effect of resveratrol on the UCP2 protein expression and ATP protein levels in hypothermic preserved rat hearts. (A) Representative immunoblot obtained with UCP2 and $\beta$-actin antibodies. (B) Densitometric analysis for the the levels of UCP2. Data are expressed as the mean \pm standard error of three experiments. (C) ATP production. Data are expressed as mean \pm standard error, $\mathrm{n}=8 .{ }^{* *} \mathrm{P}<0.01$ vs. control group; ${ }^{\# \#} \mathrm{P}<0.01$ vs. Celsior group. UCP2, uncoupling protein 2; ATP, adenosine triphosphate.
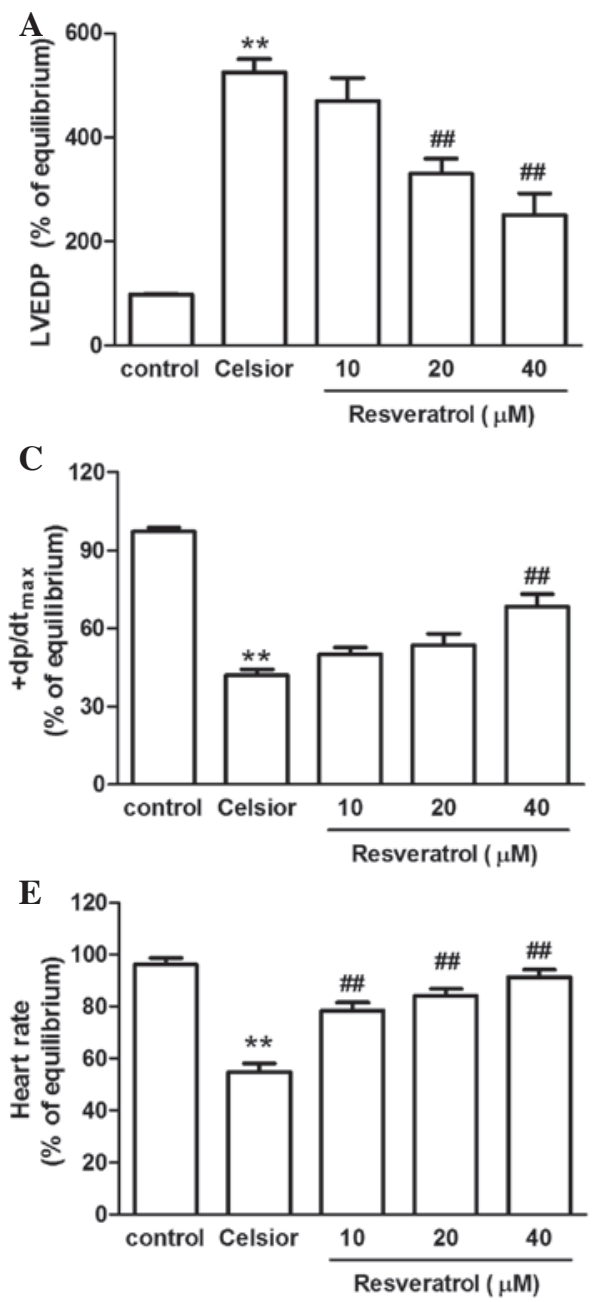

B

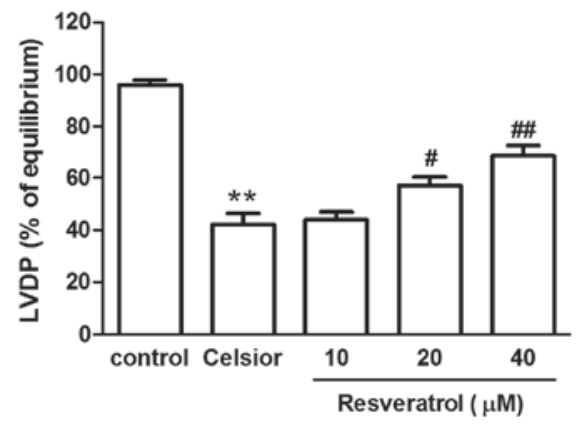

D

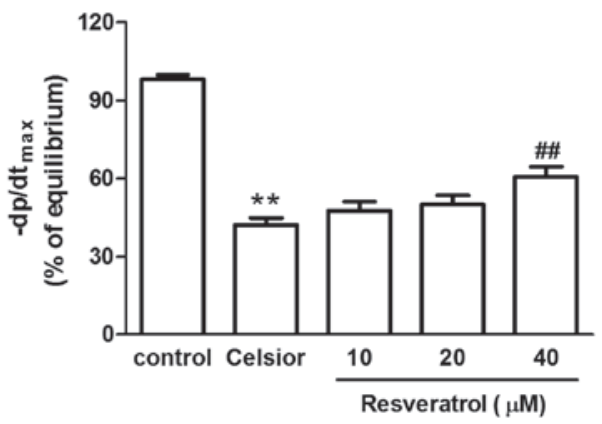

$\mathbf{F}$

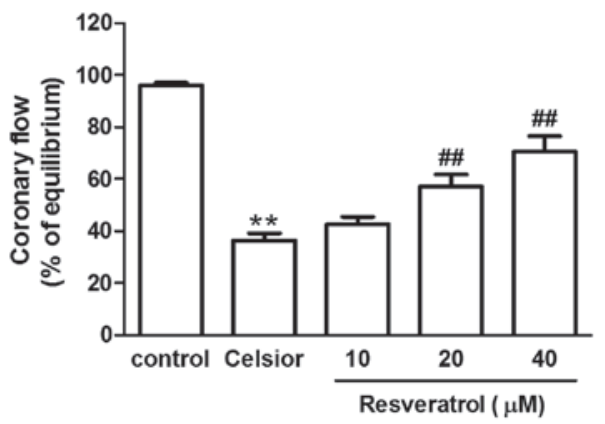

Figure 5. Effect of resveratrol on (A) LVEDP, (B) LVDP, (C) $+\mathrm{dP}^{\mathrm{dt}} \mathrm{t}_{\max }$, (D) $-\mathrm{dp}_{\mathrm{dt}} \mathrm{dt}_{\max }$, (E) heart rate and (F) coronary flow in rat hearts undergoing $9 \mathrm{~h}$ of hypothermic preservation followed by $60 \mathrm{~min}$ of reperfusion. Data are expressed as the mean \pm standard error, $\mathrm{n}=8$. ${ }^{* *} \mathrm{P}<0.01$ vs. control group; ${ }^{*} \mathrm{P}<0.05$,

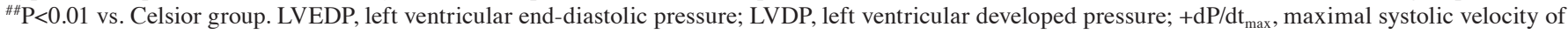
left ventricular pressure; - $\mathrm{dP} / \mathrm{dt}_{\max }$, maximal diastolic velocity of left ventricular pressure.

ROS level, suggesting that elevated UCP2 in the heart during hypothermic preservation UCP2 may result in mitochondrial damage and consequently the attenuation of ATP production. As reported, diminution of ATP synthesis is able to trigger the closing of the mitochondrial ATP-sensitive potassium channel, which is aim in cellular protection. In previous studies, Celsior solution supplemented with diazoxide significantly enhanced the LVDP recovery rate and reduced the apoptotic index (9).

There are various mechanisms whereby mitochondrial UCPs can be evoked in the context of cardiac ischemia and reperfusion (13). ROS (14), beta-adrenergic stimulation (15), 
A

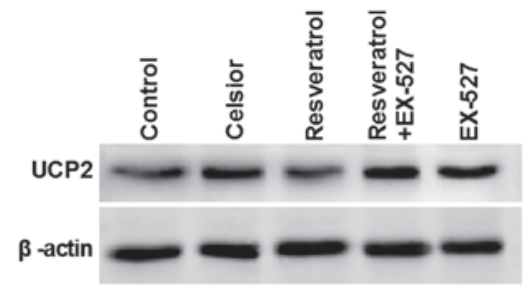

B

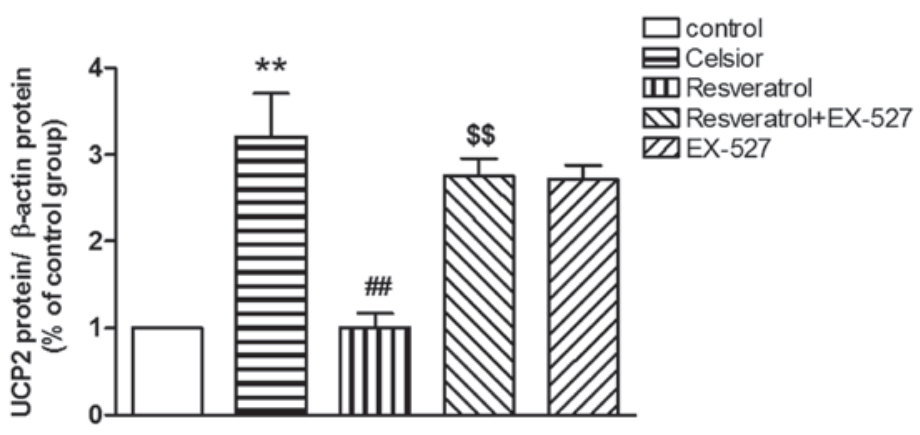

Figure 6. SIRT1 inhibitor EX-527 prevents the resveratrol-induced inhibition of UCP2 expression in hypothermic preserved hearts. (A) Representative immunoblot obtained using UCP2 and $\beta$-actin antibodies. (B) Densitometric analysis for the level of UCP2. Data are expressed as the mean \pm standard error of three experiments. ${ }^{* *} \mathrm{P}<0.01$ vs. control group (not preserved in Celsior solution); ${ }^{\# \#} \mathrm{P}<0.01$ vs. Celsior group. ${ }^{\$ \$} \mathrm{P}<0.01$ vs. resveratrol (40 $\left.\mu \mathrm{M}\right)$ group. SIRT1, silent mating type information regulation 2 homolog 1; UCP2, uncoupling protein 2.

$\square$ control $\quad$ Celsior m mesveratrol $\square$ Resveratrol+EX-527 ש EX-527

A

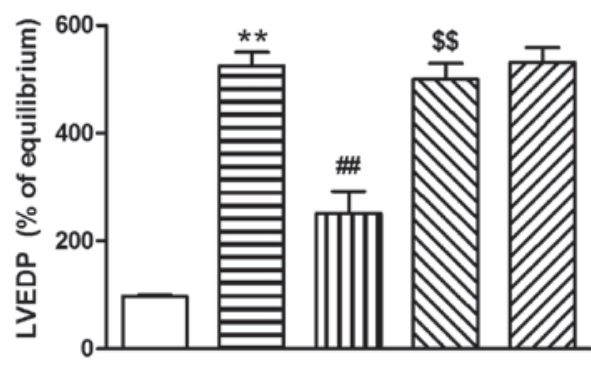

C

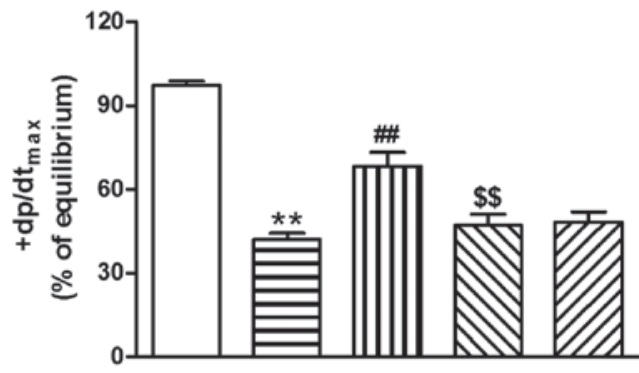

$\mathbf{E}$

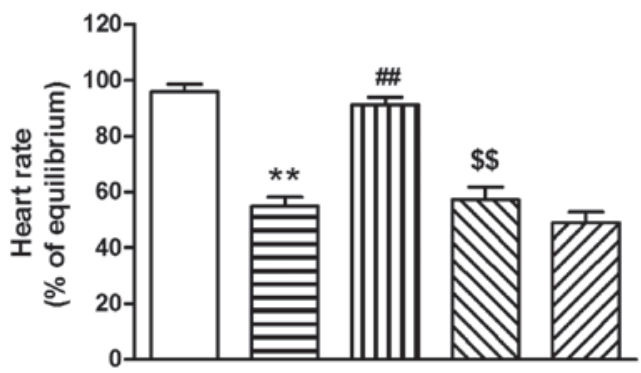

B
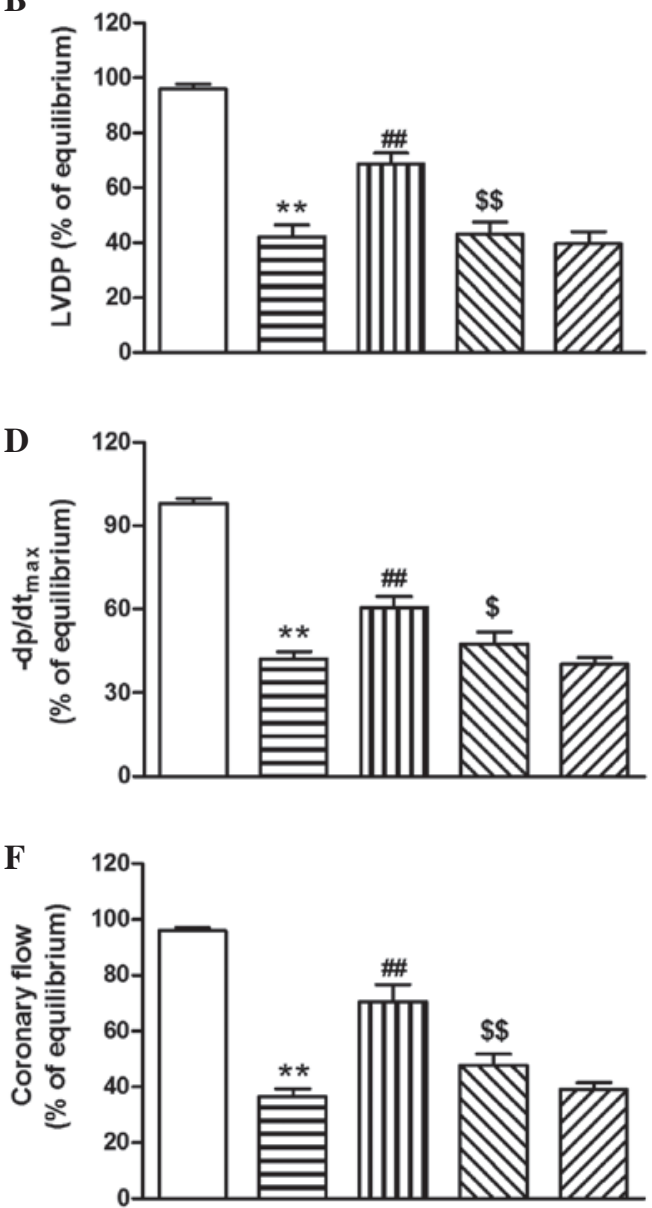

Figure 7. SIRT1 inhibitor EX-527 abolishes the resveratrol-induced improvement of (A) LVEDP, (B) LVDP, (C) +dP/dt $\max _{\max }$, (D) -dp/dt ${ }_{\max }$, (E) heart rate and (F) coronary flow in hypothermic preserved hearts. Data are expressed as the mean \pm standard error, $\mathrm{n}=8$. ${ }^{* *} \mathrm{P}<0.01$ vs. control group (not preserved in Celsior solution); ${ }^{\# \#} \mathrm{P}<0.01$ vs. Celsior group. ${ }^{\$} \mathrm{P}<0.05,{ }^{\$ \$} \mathrm{P}<0.01$ vs. resveratrol $(40 \mu \mathrm{M})$ group. SIRT1, silent mating type information regulation 2 homolog 1 ; LVEDP, left ventricular end-diastolic pressure; LVDP, left ventricular developed pressure; $+\mathrm{dP}^{-\mathrm{dt}_{\max }}$, maximal systolic velocity of left ventricular pressure; - $\mathrm{dP} / \mathrm{dt}_{\max }$, maximal diastolic velocity of left ventricular pressure. 
free fatty acids (16) and SIRT1 have been reported as regulators of UCP2 expression. SIRT1, an nicotine adenine dinucleotide(+)-dependent deacetylase, is a regulator responsible for various biological effects, predominantly in metabolism and aging (17-21). SIRT1 is essential for the maintenance of cardiac mitochondrial integrity and normal postnatal myocardium development (22). In hearts from SIRT1-deficient mice, morphological and functional mitochondrial abnormalities were observed. The expression of SIRT1 is downregulated in advanced heart failure samples compared with healthy control cardiomyocytes (23). SIRT1-dependent lysine deacetylation occurs during ischemic preconditioning and may serve a role in cardioprotective signaling (24). Downstream targets of SIRT1 include peroxisome proliferator-activated receptor (PPAR)- $\gamma$, PPAR $\gamma$ coactivator- $1 \alpha$ and UCP2 (25). Resveratrol is a natural polyphenolic compound that has cardioprotective, anticancer and anti-inflammatory properties $(26,27)$, through a SIRT1-dependent (28) or independent pathway (29). It was identified that the SIRT1 protein expression in rat hearts reduced following hypothermic preservation, while Celsior solution supplemented with the SIRT1 activator resveratrol inhibited the UCP2 protein overexpression, prevented the decline in ATP production, improving cardiac function. The SIRT1 inhibitor EX-527 abolished the resveratrol-induced inhibition of UCP2 overexpression and cardiac protection in the hypothermic preserved rat heart. Thus, it was suggested that SIRT1 is required for cardioprotection against ischemia-reperfusion injury, and reduction of SIRT1 is associated with overexpression of UCP2. In the kidney and brain, SIRT1 is suggested to regulate the mitochondrial UCP2 via the peroxisome proliferator-activated receptor- $\gamma$ coactivator-1 $\alpha /$ PPAR $\alpha$ signaling pathway $(30,31)$.

In conclusion, these results suggest that downregulation of UCP2 expression in the hypothermic preserved rat heart in part initiated the protection mechanism via the SIRT1 pathway.

\section{Acknowledgements}

The current study was supported by the National Natural Science Foundation of China (grant no. 81270178), the Science and Technology Department of Zhejiang Province (grant no. 2015C37129), and Jiaxing Science and Technology Project (grant no. 2012AY1075-5).

\section{References}

1. Shah KB and Parameshwar J: Advances in heart transplantation: The year in review. J Heart Lung Transplant 30: 241-246, 2011.

2. Cannon RM, Hughes MG, Jones CM, Eng M and Marvin MR: A review of the United States experience with combined heart-liver transplantation. Transpl Int 25: 1223-1228, 2012.

3. Baines CP: The cardiac mitochondrion: Nexus of stress. Annu Rev Physiol 72: 61-80, 2010.

4. Alán L, Smolková K, Kronusová E, Santorová J and Jezek P: Absolute levels of transcripts for mitochondrial uncoupling proteins UCP2, UCP3, UCP4 and UCP5 show different patterns in rat and mice tissues. J Bioenerg Biomembr 41: 71-78, 2009.

5. McLeod CJ, Aziz A, Hoyt RF Jr, McCoy JP Jr and Sack MN: Uncoupling proteins 2 and 3 function in concert to augment tolerance to cardiac ischemia. J Biol Chem 280: 33470-33476, 2005 .
6. Zungu M, Alcolea MP, Garcia-Palmer FJ, Young ME and Essop MF: Genomic modulation of mitochondrial respiratory genes in the hypertrophied heart reflects adaptive changes in mitochondrial and contractile function. Am J Physiol Heart Circ Physiol 293: H2819-H2825, 2007.

7. National Research Council (US) Committee for the Update of the Guide for the Care and Use of Laboratory Animals: Guide for the Care and Use of Laboratory Animals. 8th edition. National Academies Press, Washington, D.C., 2011.

8. Yan ZK, Hu ZB, Pan XH, Chen YY, Zhang XM and Shen YL: Diazoxide supplemented Celsior solution improves hypothermic heart preservation effect in rat through activation of mitochondrial ATP-sensitive potassium channel. Pharm Biol 47: 1060-1066, 2009.

9. Yang F, Chen WL, Zheng MZ, Yu GW, Xu HJ, Shen YL and Chen YY: Heat shock protein 90 mediates anti-apoptotic effect of diazoxide by preventing the cleavage of Bid in hypothermic preservation rat hearts. J Heart Lung Transplant 30: 928-934, 2011.

10. Zhou HY, Zhang LN, Zheng MZ, Wang LL, Chen YY and Shen YL: Improved myocardial function with supplement of levosimendan to celsior solution. J Cardiovasc Pharmacol 64: 256-265, 2014.

11. Teshima Y, Akao M, Jones SP and Marbán E: Uncoupling protein-2 overexpression inhibits mitochondrial death pathway in cardiomyocytes. Circ Res 93: 192-200, 2003.

12. Bodyak N, Rigor DL, Chen YS, Han Y, Bisping E, Pu WT and Kang PM: Uncoupling protein 2 modulates cell viability in adult rat cardiomyocytes. Am J Physiol Heart Circ Physiol 293: H829-H835, 2007.

13. Toda $\mathrm{C}$ and Diano S: Mitochondrial UCP2 in the central regulation of metabolism. Best Pract Res Clin Endocrinol Metab 28: 757-764, 2014.

14. Brand MD, Affourtit C, Esteves TC, Green K, Lambert AJ, Miwa S, Pakay JL and Parker N: Mitochondrial superoxide: Production, biological effects and activation of uncoupling proteins. Free Radic Biol Med 37: 755-767, 2004.

15. Ishizawa $M$, Mizushige $K$, Noma $T$, Namba $T$, Guo $P$, Murakami K, Tsuji T, Miyatake A, Ohmori K and Kohno M: An antioxidant treatment potentially protects myocardial energy metabolism by regulating uncoupling protein 2 expression in a chronic beta-adrenergic stimulation rat model. Life Sci 78: 2974-2982, 2006.

16. Murray AJ, Panagia M, Hauton D, Gibbons GF and Clarke K: Plasma free fatty acids and peroxisome proliferator-activated receptor alpha in the control of myocardial uncoupling protein levels. Diabetes 54: 3496-3502, 2005.

17. Yacoub R, Lee K and He JC: The Role of SIRT1 in Diabetic Kidney Disease. Front Endocrinol (Lausanne) 5: 166, 2014.

18. Rehan L, Laszki-Szcząchor K, Sobieszczańska M and Polak-Jonkisz D: SIRT1 and NAD as regulators of ageing. Life Sci 105: 1-6, 2014.

19. Philp A and Schenk S: Unraveling the complexities of SIRT1-mediated mitochondrial regulation in skeletal muscle. Exerc Sport Sci Rev 41: 174-181, 2013.

20. Kitada M and Koya D: SIRT1 in type 2 diabetes: Mechanisms and therapeutic potential. Diabetes Metab J 37: 315-325, 2013.

21. Sundaresan NR, Pillai VB and Gupta MP: Emerging roles of SIRT1 deacetylase in regulating cardiomyocyte survival and hypertrophy. J Mol Cell Cardiol 51: 614-618, 2011.

22. Planavila A, DominguezE, Navarro M, Vinciguerra M,Iglesias R, Giralt M, Lope-Piedrafita S, Ruberte J and Villarroya F: Dilated cardiomyopathy and mitochondrial dysfunction in Sirtl-deficient mice: A role for Sirt1-Mef2 in adult heart. J Mol Cell Cardiol 53: 521-531, 2012.

23. Lu TM, Tsai JY, Chen YC, Huang CY, Hsu HL, Weng CF, Shih CC and Hsu CP: Downregulation of Sirtl as aging change in advanced heart failure. J Biomed Sci 21: 57, 2014.

24. Nadtochiy SM, Yao H, McBurney MW, Gu W, Guarente L, Rahman I and Brookes PS: SIRT1-mediated acute cardioprotection. Am J Physiol Heart Circ Physiol 301: H1506-H1512, 2011.

25. Chaudhary N and Pfluger PT: Metabolic benefits from Sirt1 and Sirtl activators. Curr Opin Clin Nutr Metab Care 12: 431-437, 2009.

26. Pangeni R, Sahni JK, Ali J, Sharma S and Baboota S: Resveratrol: Review on therapeutic potential and recent advances in drug delivery. Expert Opin Drug Deliv 11: 1285-1298, 2014.

27. Granzotto A and Zatta P: Resveratrol and Alzheimer's disease: Message in a bottle on red wine and cognition. Front Aging Neurosci 6: 95, 2014. 
28. Zong Y, Sun L, Liu B, Deng YS, Zhan D, Chen YL, He Y, Liu J, Zhang ZJ, Sun J and Lu D: Resveratrol inhibits LPS-induced MAPKs activation via activation of the phosphatidylinositol 3-kinase pathway in murine RAW 264.7 macrophage cells. PLoS One 7: e44107, 2012.

29. Centeno-Baez C, Dallaire P and Marette A: Resveratrol inhibition of inducible nitric oxide synthase in skeletal muscle involves AMPK but not SIRT1. Am J Physiol Endocrinol Metab 301: E922-E930, 2011.
30. Rubattu S, Di Castro S, Cotugno M, Bianchi F, Mattioli R, Baima S, Stanzione R, Madonna M, Bozzao C, Marchitti S, et al: Protective effects of Brassica oleracea sprouts extract toward renal damage in high-salt-fed SHRSP: Role of AMPK/PPAR $\alpha / \mathrm{UCP} 2$ axis. J Hypertens 33: 1465-1479, 2015.

31. Wang SJ, Zhao XH, Chen W, Bo N, Wang XJ, Chi ZF and Wu W: Sirtuin 1 activation enhances the PGC-1/mitochondrial antioxidant system pathway in status epilepticus. Mol Med Rep 11: 521-526, 2015. 